\title{
Shensong Yangxin capsule reduces atrial fibrillation susceptibility by inhibiting atrial fibrosis in rats with post-myocardial infarction heart failure
}

This article was published in the following Dove Press journal: Drug Design, Development and Therapy

Jin Ma, ${ }^{1, *}$ Chunxia Yin, ${ }^{1, *}$ Shiyu Ma, ${ }^{2}$ Huiliang Qiu, ${ }^{1}$ Chaoyang Zheng,' Qiuxiong Chen,' Chunhua Ding, ${ }^{1,3}$ Weihui Lv'

'Heart Center, Guangdong Provincial Hospital of Chinese Medicine, 2nd Affiliated Hospital of Guangzhou University of Chinese Medicine, Guangzhou 510006, China;

${ }^{2}$ Department of Critical-Care Medicine, Guangdong Provincial Hospital of Chinese Medicine, 2nd Affiliated Hospital of Guangzhou University of Chinese Medicine, Guangzhou 5I0006, China; ${ }^{3} \mathrm{Cardiac}$ Department, Aerospace Center Hospital, Peking University Aerospace Clinical College of Medicine, Beijing I00049, China

*These authors contributed equally to this work

Correspondence: Weihui Lv Heart Center, Guangdong Provincial Hospital of Chinese Medicine, 2nd Affiliated Hospital of Guangzhou University of Chinese Medicine, 55 Neihuan Xilu, Panyu District, Guangzhou 510006, China

Tel +86 I39 24l3 2258

Fax +8602039378372

Email drlvwh@।63.com

Chunhua Ding

Cardiac Department, Aerospace Center Hospital, Peking University Aerospace Clinical College of Medicine, 15 Yuquan Road, Haidian District, Beijing 100049, China

Tel +86 I86 I2II 7275

Fax +86 0105997 I 397

Email dingmd@gmail.com
Purpose: Shensong Yangxin (SSYX) capsule is a traditional Chinese medicine that has been used widely to treat cardiac arrhythmia. This study aimed to assess whether SSYX prevents atrial fibrillation (AF) after chronic myocardial infarction (MI)-induced heart failure and to determine the underlying mechanisms.

Materials and methods: The study included 45 male Sprague Dawley rats. The rats underwent MI induction or sham surgery. One week after MI induction surgery, we performed serial echocardiography and administered SSYX capsule to some rats that experienced MI. After 4 weeks of treatment, AF inducibility was assessed with transesophageal programmed electrical stimulation technology. Additionally, multielectrode array assessment, histological analysis, and Western blot analysis were performed.

Results: AF inducibility was significantly lower in SSYX rats than in MI rats (33.3\% vs 73.3\%, $P<0.05$ ). Additionally, conduction velocities in the left atrium were greater in SSYX rats than in MI rats. Moreover, SSYX decreased left atrial fibrosis, downregulated TGF- $\beta 1$, MMP-9, TIMP-I, and type I and III collagen expressions, and inhibited the differentiation of cardiac fibroblasts to myofibroblasts.

Conclusion: SSYX reduces AF inducibility after MI by improving left atrial conduction function via the inhibition of left atrial fibrosis. It prevents the development of an MI-induced vulnerable substrate for AF.

Keywords: Shensong Yangxin capsule, atrial fibrillation, fibrosis, electrophysiology, heart failure

\section{Introduction}

Atrial fibrillation (AF) is a common, persistent cardiac arrhythmia and is associated with remarkably increased rates of hospitalization, morbidity, and mortality. ${ }^{1,2}$ Despite clinical and fundamental research efforts over the past few decades, the mechanisms underlying $\mathrm{AF}$ remain unclear. AF is complex arrhythmia originating from an abnormal atrial substrate and has various predisposing factors, such as heart failure (HF), diabetes, ischemic heart disease, aging, hypertension, obesity, obstructive sleep apnea, genetic factors, and cardiorespiratory fitness. ${ }^{3,4}$ The abnormal atrial substrate for AF involves electrical, structural, and ionic changes, and this phenomenon is termed as "atrial remodeling".

Oxidative stress and inflammation play important roles in the promotion of atrial remodeling, leading to atrial ectopy, atrial interstitial fibrosis, and increased risk of stroke. ${ }^{3,5}$ A reasonable amount of experimental and clinical evidence indicates that atrial fibrosis plays an important role in AF. ${ }^{6,7}$ Atrial fibrosis occurs when fibroblasts 
differentiate to myofibroblasts in the myocardium, resulting in collagen deposition. TGF- $\beta 1$ plays a vital role in collagen synthesis and is expressed at high levels in the fibrotic cardiac tissue. Progressive accumulation of the fibrous tissue in the myocardium is one of the major aspects of cardiac structural remodeling. The formation and redistribution of the fibrous tissue modulate myocardial geometry to adapt to new conditions of pathophysiological functioning. This adaptation process involves the extracellular matrix (ECM), which contains large amounts of fibers and collagen. The normal cardiac ECM plays an important role in maintaining homeostasis between matrix protein synthesis and degradation. However, excessive ECM production commonly results in abnormalities in cardiac contraction and relaxation. ${ }^{8}$ Drugs that prevent myocardial fibrosis and improve heart function might be useful for AF. In recent years, accumulating experimental evidence has suggested that the inhibition of atrial fibrosis using drugs, such as eplerenone, ${ }^{9}$ aliskiren, ${ }^{10}$ and taurine, ${ }^{11}$ effectively inhibits AF susceptibility in patients and animal models.

The traditional Chinese medicine, Shensong Yangxin (SSYX) capsule, has been widely used to treat cardiac arrhythmia. It consists of 12 ingredients (Table 1), and its use has been approved by the State Food and Drug Administration of China in 2003 (File No Z20030058). It is used in the treatment of atrial premature complexes and ventricular premature complexes in China. ${ }^{12,13}$ Clinical studies have demonstrated that SSYX capsule can effectively reduce paroxysmal AF. ${ }^{14}$ Furthermore, previous studies by patch clamp have suggested that SSXY capsule can inhibit multiple ion channels related to AF. ${ }^{15,16}$ Preliminary studies have suggested that SSYX capsule could inhibit ventricular fibrosis and improve cardiac function in diabetic rats. ${ }^{17}$ However, the effect of SSYX on atrial fibrosis remains unclear. The antifibrosis effect of SSYX in the atria may be a potential mechanism for treating AF. The present study aimed to determine the effect of SSYX on atrial fibrosis in myocardial infarction (MI)-induced HF rats and to explore the underlying molecular mechanisms.

\section{Materials and methods Shensong Yangxin}

SSYX capsule was provided by Yiling Pharmaceutical Corporation (Lot No 1508040; Shijiazhuang, China). The contents of SSYX capsule and their proportions are shown in Table 1. The quality of SSYX capsule was confirmed by ultrahigh performance liquid chromatography combined with quadrupole time-of-flight mass spectrometry, as previously described. $^{18}$

\section{Animal model}

Male Sprague Dawley rats (weight, 250-280 g) were obtained from the Animal Center of Southern Medical University. All experimental procedures were performed in strict accordance with the Guide for the Care and Use of Laboratory Animals published by the US National Institutes of Health (publication number 85-23, revised 1996), and all studies were approved by the Animal Care Committee of Guangdong Provincial Hospital of Chinese Medicine (No 2016044). The rats underwent MI establishment surgery or sham surgery. MI was established by ligating the coronary artery, as previously described. ${ }^{19}$ Briefly, rats were anesthetized, intubated, and then ventilated (Harvard

Table I Herbs of SSYX

\begin{tabular}{|c|c|c|c|c|c|}
\hline Chinese name & Botanical name & The part used & Origin & Ratio & Voucher numbers \\
\hline Ren Shen & Panax ginseng C.A. Mey. & Root & Jilin & 2 & 120103003 \\
\hline Mai Dong & Ophiopogon japonicus (Thunb.) Ker Gawl. & Tuber & Sichuan & 2.5 & 120124056 \\
\hline Gan Song & Nardostachys grandiflora DC. & Root & Sichuan & 2 & 120124054 \\
\hline Sang Ji Sheng & Taxillus estipitatus (Stapf) Danser & Stem & Guangxi & 4.2 & 120124058 \\
\hline Suan Zao Ren & Ziziphus jujuba Mill. & Fruit & Hebei & 4.2 & 120124007 \\
\hline Shan Zhu Yu & Cornus officinalis Siebold \& Zucc. & Fruit & Shanxi & 5 & 120124059 \\
\hline Dan Shen & Salvia miltiorrhiza Bunge & Root & Shangdong & 5 & 120124053 \\
\hline Chi Shao & Paeonia lactiflora Pall. & Root & Hebei & 2 & 120124004 \\
\hline Wu Wei Zi & Schisandra sphenanthera Rehder \& E.H. Wilson & Fruit & Henan & 1.5 & 120124057 \\
\hline Long Gu & Polypodium pseudo-amoenum Ching & Skeleton & Gansu & 3.3 & 120124060 \\
\hline Tu Bie Chong & Eupolyphaga sinensis Walker & Whole animal & Hebei & 1.7 & 120101201 \\
\hline Huang Lian & Coptis chinensis Franch. & Root & Sichuan & I & 120124055 \\
\hline
\end{tabular}

Abbreviation: SSYX, Shensong Yangxin. 
Apparatus Co., South Natick, MA, USA) at a respiratory rate of 65 cycles/min. After thoracotomy was performed to expose the heart, the left coronary artery was permanently ligated with a 6-0 nylon silk suture placed 2-3 mm from the tip of the left auricle. An MI model is successfully established when the left ventricle anterior wall turns pallor and the ST segment appears to be elevated on electrocardiography. The sham-operated rats $(n=15)$ underwent the same procedure without ligation of the left coronary artery. In rats that underwent the MI establishment surgery, echocardiography was performed 1 week postoperatively, and only rats with a left ventricular ejection fraction $(\mathrm{EF})$ of $<45 \%$ were considered for further experiments. The selected rats were randomly divided into the following two groups: MI $(n=15)$ and SSYX ( $\mathrm{n}=15)$ groups. Rats in the SSYX group received $600 \mathrm{mg} / \mathrm{kg}$ (according to clinical dose) SSYX for 4 weeks, whereas those in the sham and MI groups received the same dose of water for 4 weeks.

\section{Echocardiography}

Serial echocardiography was performed using a highresolution echocardiography system (Vevo 2100; VisualSonics Inc., Toronto, ON, Canada) at 1 and 5 weeks after MI, respectively. All the 45 rats were anesthetized with isoflurane. Transthoracic echocardiography was performed with a $21 \mathrm{MHz}$ phased array probe to measure EF and fractional shortening using M-mode recordings obtained at the level of the papillary muscles. Each echocardiographic variable was determined in at least four separate left ventricular images of the same heart.

\section{Programmed electrical stimulation and induction of $A F$}

AF was defined as irregular, atrial arrhythmia and rapid atrial activation with varying electrographic morphologies lasting for $\geq 2$ seconds. ${ }^{20}$ After the induction of anesthesia, the tracheal cannula was intubated via the mouth and mechanical ventilation was initiated with a small animal ventilator (Harvard Apparatus Co.). For atrial stimulation, a 4-French quadripolar catheter was advanced through the esophagus and placed at the site with the lowest threshold for atrial capture. The inducibility of atrial arrhythmia was tested using the automated stimulator software by applying 35 -second bursts. Each burst had a cycle length of $20 \mathrm{~ms}$ and pulse width of $5 \mathrm{~ms}$. AF was considered in case of rapid, irregular atrial rhythm lasting for at least 2 seconds following burst pacing. AF was considered inducible if $\geq 1$ burst in two series of bursts evoked an AF episode in rats. AF inducibility and duration were recorded and analyzed.

\section{Multielectrode array (MEA) measurements}

MEA technology was utilized in vitro 4 weeks after SSYX capsule administration. After the induction of anesthesia, the heart was immediately removed, and the left atrium was excised from the isolated heart and immersed in modified Tyrode's solution (composition in mmol/L: $\mathrm{NaCl}, 130$; $\mathrm{NaHCO}_{3}, 20 ; \mathrm{NaH}_{2} \mathrm{PO}_{4}, 1.2 ; \mathrm{MgCl}_{2}, 1 ; \mathrm{KCl}, 4.0$; glucose, 5.6; $\mathrm{CaCl}_{2}, 1.8 ; 2,3$-butanedione monoxime, $15 ; \mathrm{pH}$ 7.4). An MEA dish (Multi Channel Systems, Reutlingen, Germany) containing 120 tipped platinum electrodes with an electrode height between 25 and $35 \mu \mathrm{m}$ and interelectrode distance of $100 \mu \mathrm{m}$ was used. The electrode arrays were mounted onto a printed circuit board and were then fitted into the MEA 2100 System interface (Multi Channel Systems). The atrium was positioned at the center of the MEA dish, kept in contact with the 120 electrodes using a slice holder, and continuously superfused with oxygenated modified Tyrode's solution at $37^{\circ} \mathrm{C}$. Electrical stimulation (bipolar pulses, 1-7 V; duration, $1,000 \mu \mathrm{s})$ was applied via one of the MEA microelectrodes. Data were sampled at $10 \mathrm{kHz}$ per channel with simultaneous data acquisition using Cardio2D+ software (Multi Channel Systems). Five fields were recorded in each atrium. All data were analyzed to generate activation maps and measure the conduction velocity $(\mathrm{CV})$.

\section{Masson's trichrome staining}

Atrial tissue samples were fixed in 4\% paraformaldehyde for 24 hours. The left atrium was then cut into two half longitudinal sections. After standard paraffin embedding, Masson's trichrome staining was performed. The total left atrial fibrosis area (\%) was calculated using cellSens Viewer software (Olympus Imaging Corp., Tokyo, Japan) to evaluate left atrial fibrosis in each group.

\section{Western blot analysis}

Total protein was obtained from the left atrium, subjected to standard procedures, and its concentration was determined using the bicinchoninic acid protein assay kit (Thermo Fisher Scientific, Waltham, MA, USA). Protein samples were separated using 10\% SDS-PAGE and transferred onto a polyvinylidene difluoride membrane. The membrane was blocked with 5\% skimmed milk for 1 hour at room temperature and was incubated with primary antibodies overnight at $4^{\circ} \mathrm{C}$. The diluted concentrations of the primary antibodies 
(Abcam, Cambridge, UK; Santa Cruz Biotechnology; or Cell Signaling Technology) were as follows: collagen I, 1:500; collagen III, 1:500; $\alpha$-smooth muscle actin ( $\alpha$-SMA), 1:1,000; TGF- $\beta 1,1: 1,000$; mothers against decapentaplegic homolog 3 (Smad3), 1:500; matrix metalloproteinase 9 (MMP-9), 1:500; tissue inhibitor of metalloproteinase 1 (TIMP-1), 1:500; and GAPDH, 1:1,000. The secondary antibodies (Cell Signaling Technology) were horseradish peroxidase-labeled antibodies diluted at 1:3,000. The membrane was incubated with secondary antibodies for 1 hour at $25^{\circ} \mathrm{C}$. Protein bands were visualized using Enhanced Chemiluminescence Plus (EMD Millipore, Billerica, MA, USA). GAPDH was used as the internal control. The final results were expressed as fold changes by normalizing the data to control values.

\section{Data analysis and statistics}

Data are expressed as the mean $\pm \mathrm{SD}$, except AF duration, which is expressed as the median and IQR (25\%-75\%). AF inducibility comparisons between the control and SSYX groups were performed using the Fisher's exact test. Comparisons between groups were performed with ANOVA followed by the Bonferroni post-test, and the least significant difference method was used for post hoc multiple comparisons. All statistical analyses were performed using SPSS version 13.0 software (SPSS Inc., Chicago, IL, USA). A $P$-value $<0.05$ was considered statistically significant.

\section{Results \\ SSYX attenuated the inducibility and duration of $A F$}

Figure $1 \mathrm{~A}$ shows a representative electrocardiogram of atrial burst stimulation-induced AF. Before the burst, the rat was in sinus rhythm (Figure 1Aa). After termination of the burst, the rat was in irregular atrial rhythm with irregular ventricular response (Figure $1 \mathrm{Ab}$ ). The $\mathrm{AF}$ episode terminated spontaneously within a few seconds and sinus rhythm resumed (Figure 1Ac). In contrast, a noninduced rat continued to show sinus rhythm after the burst (Figure 1Ad). The rate of AF inducibility was markedly higher in the MI group (73.3\%) than in the sham group (6.67\%) and markedly lower in the SSYX group (33.3\%) than in the MI group (Figure 1B). Furthermore, the mean AF episode duration was significantly higher in the MI group (251-1,060 seconds) than in the sham group (11.7-47.8 seconds) and significantly lower in the SSYX group (52.5-303.8 seconds) than in the MI group $(P<0.05$; Figure 1C).

\section{SSYX improved cardiac function and atrial conduction function after $\mathrm{MI}$}

Cardiac function was evaluated with echocardiography. Echocardiography was performed 4 weeks after SSYX administration. Representative M-mode echocardiograms for the groups are presented in Figure 2A. EF\% and fraction shortening (\%) were significantly lower in the MI group $(37.8 \% \pm 3.1 \%$ and $19.2 \% \pm 1.7 \%$, respectively) than in the sham group $(76.5 \% \pm 7.1 \%$ and $41.2 \% \pm 4.3 \%$, respectively) and higher in the SSYX group (46.4\% $\pm 6.2 \%$ and $24.5 \% \pm 3.8 \%$, respectively) than in the MI group $(P<0.05$; Figure 2B and C).

Left atrial surface conduction activation maps (Figure 3A) and CV maps (Figure 3B) demonstrated crowding of isochrones and a slower $\mathrm{CV}$ in the MI group $(13.5 \pm 3.7 \mathrm{~cm} / \mathrm{s})$ than that in the sham group $(35.1 \pm 2.5 \mathrm{~cm} / \mathrm{s}, P<0.01)$, in addition to heterogeneous conduction. On the other hand, left atrial $\mathrm{CV}$ was higher $(P<0.01$; Figure $3 \mathrm{C})$ and homogeneity was better in the SSYX group than in the MI group.

\section{SSYX inhibited left atrial interstitial fibrosis}

Collagen production and deposition were evaluated using Masson's trichrome staining and Western blot analysis. Fibrosis-positive areas in the left atrium were more common in the MI group than in the sham group (Figure 4A) and less common in the SSYX group than in the MI group $(P<0.05$; Figure 4B). Type I and III collagen expressions were examined using Western blot analysis and were found to be significantly higher in the MI group than in the sham group and markedly lower in the SSYX group than in the MI group $(P<0.05$; Figure $4 \mathrm{C}$ and $\mathrm{D})$.

\section{SSYX inhibited the differentiation of cardiac fibroblasts to myofibroblasts}

$\alpha$-SMA is a marker for the differentiation of fibroblasts to myofibroblasts. Therefore, we used Western blot analysis to assess the role of SSYX in the differentiation of fibroblasts to myofibroblasts. $\alpha$-SMA expression was significantly higher in the MI group than in the sham group. Interestingly, $\alpha$-SMA expression was significantly lower in the SSYX group than in the MI group ( $P<0.05$; Figure 5A). The TGF- $\beta 1$ signaling pathway has been reported to be important for fibrosis progression in various organs. In the present study, we explored whether SSYX could attenuate fibrosis through the TGF- $\beta 1$ signaling pathway. Western blot analysis indicated that TGF- $\beta 1$ expression was significantly 
A a

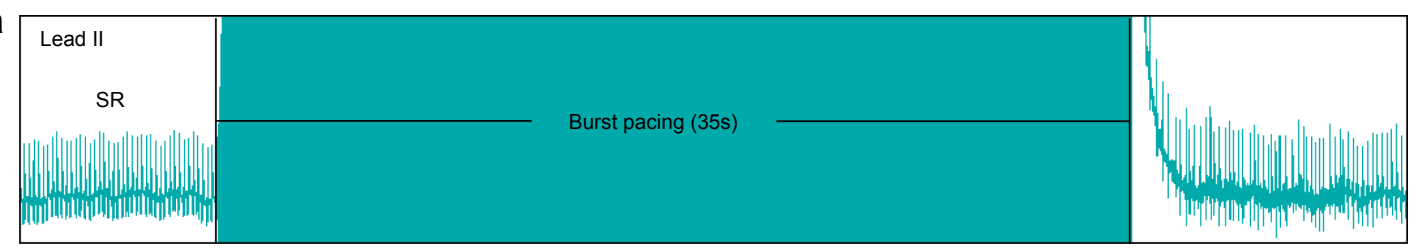

b

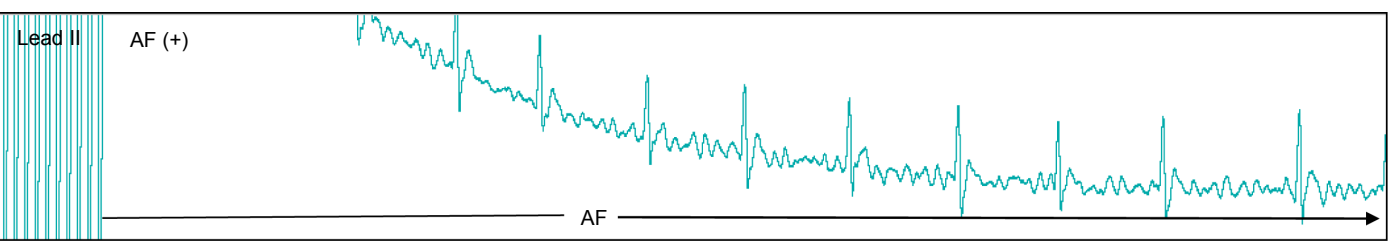

C

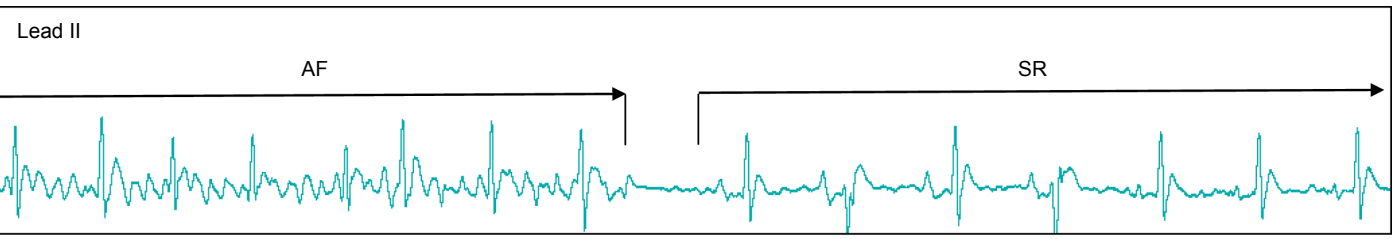

d ||l|Lead III|| AF $(-)$

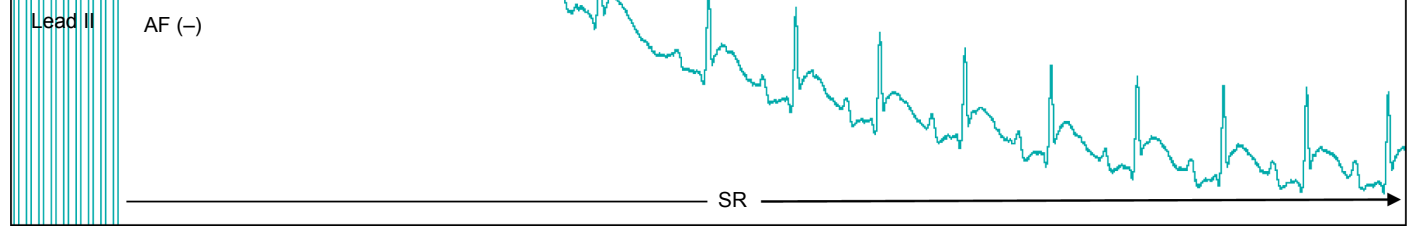

e
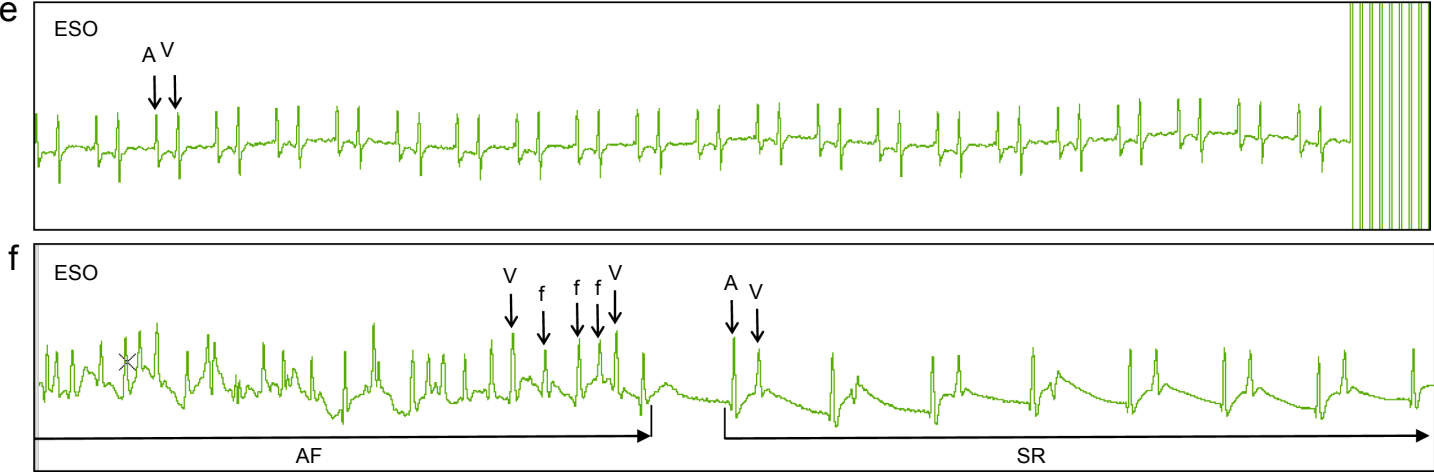

B

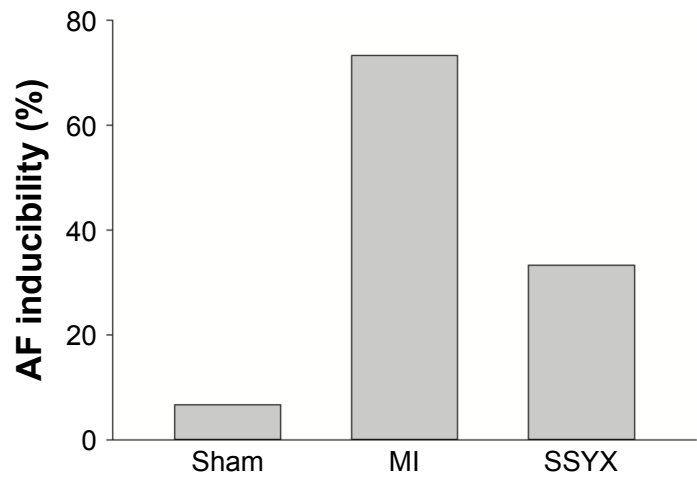

C

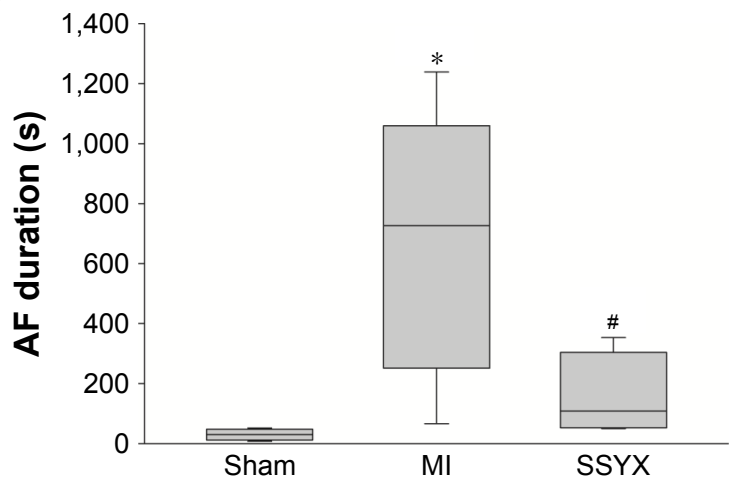

Figure I SSYX inhibited AF inducibility and duration after MI.

Notes: (A) ECG recorded after 4 weeks. (a) An example of an induced AF episode. Before the burst, the rat was in SR. After termination of the burst (b), the rat displayed an irregular atrial rhythm with an irregular ventricular response. After a few seconds (c), the AF episode terminated spontaneously and SR resumed. (d) An example of a noninduced AF episode. After termination of the burst, the rat also displayed SR. (e) Esophageal ECG which recorded clear, high, and bidirectional atrial wave that was used to help to determine the position of the electrode and distinguish SR or AF. ( $f$ ) A pause representing the termination and cardioversion of AF recorded by esophageal ECG. (B) Bar graphs indicate AF inducibility. SSYX inhibited AF inducibility in rats compared to $M I$. (C) Bar graphs indicate AF duration. SSYX decreased AF episode duration. $* P<0.05$ vs sham rats; ${ }^{\#}<<0.05$ vs sham rats.

Abbreviations: A, atrial depolarization; $\mathrm{V}$, ventricular depolarization; f, f-wave; AF, atrial fibrillation; ECG, electrocardiogram; ESO, esophageal electrocardiogram; MI, myocardial infarction; SR, sinus rhythm; SSYX, Shensong Yangxin. 
A
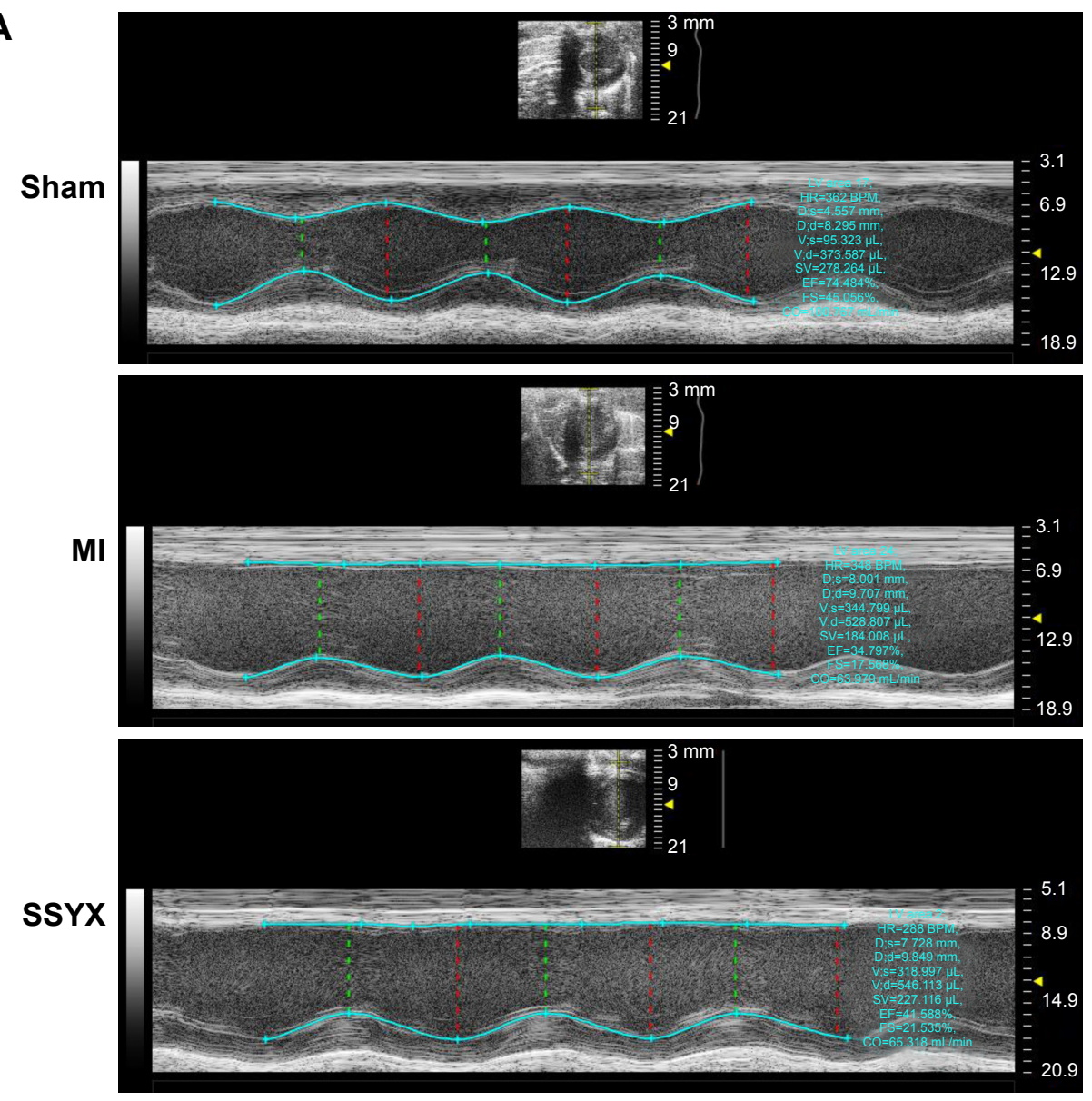

B

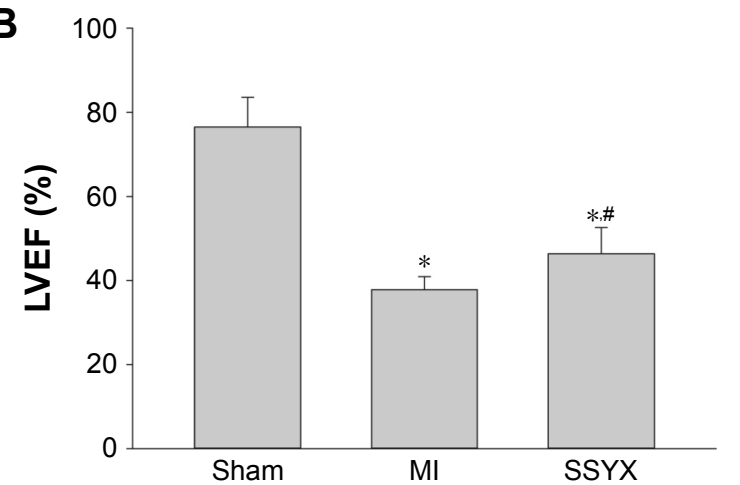

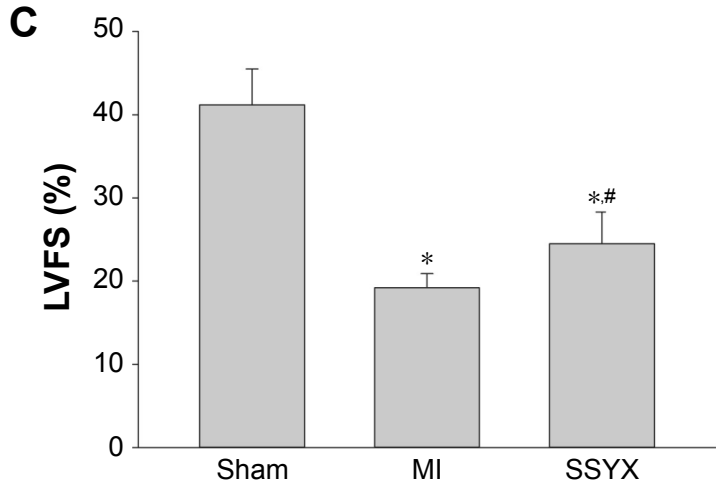

Figure 2 SSYX improved the cardiac function after MI.

Notes: (A) Representative examples of M-mode echocardiogram of three groups. (B) Bar graphs indicate the EF. SSYX improved the EF after MI. (C) Bar graphs indicate the FS. SSYX improved the FS after MI. ${ }^{* P}<0.05$ vs sham rats; ${ }^{*} P<0.05$ vs sham rats.

Abbreviations: EF, ejection fraction; FS, fraction shortening; LV, left ventricular; LVEF, left ventricular ejection fraction; LVFS, left ventricular fraction shortening; MI, myocardial infarction; SSYX, Shensong Yangxin.

higher in the MI group than in the sham group and lower in the SSYX group than in the MI group ( $P<0.05$; Figure 5B). However, Smad3 expression did not differ among the sham, MI, and SSYX groups ( $P>0.05$; Figure 5C).

\section{SSYX downregulated MMP-9 and TIMP-I expressions}

MMPs are proteolytic enzymes involved in the degradation of collagen and ECM components. ${ }^{10-14}$ TIMP maintains the homeostatic balance of myocardial ECM by inhibiting activated matrix MMPs. MMP and TIMP expressions are consistently upregulated in myocardial fibrosis; hence, these proteins can be used as markers for fibrosis. We, therefore, investigated the effect of SSYX on MMP-9 and TIMP-1 expressions. Western blot analysis showed that MMP-9 and TIMP-1 expressions were significantly higher in the MI group than in the sham group and significantly lower in the SSYX group than in the MI group (Figure 5D and $\mathrm{E} ; P<0.05$ ). 
A

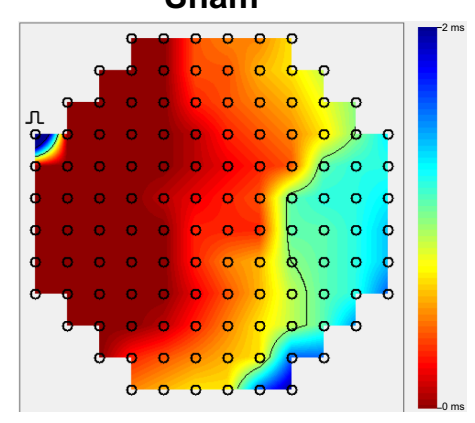

B



MI
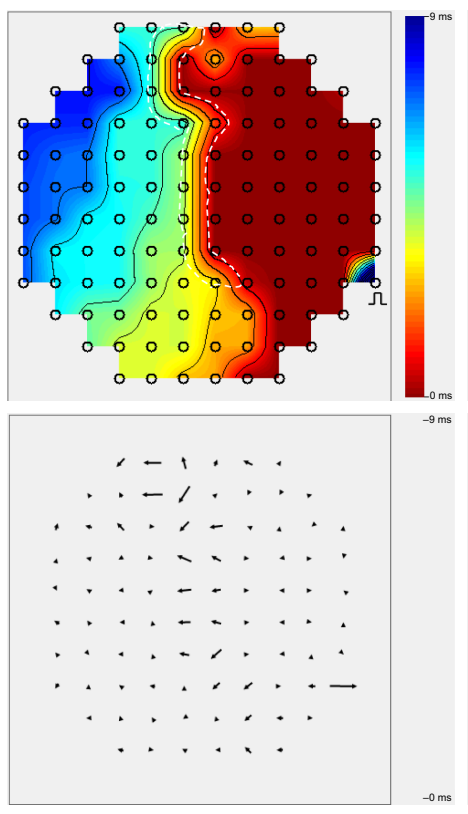

SSYX
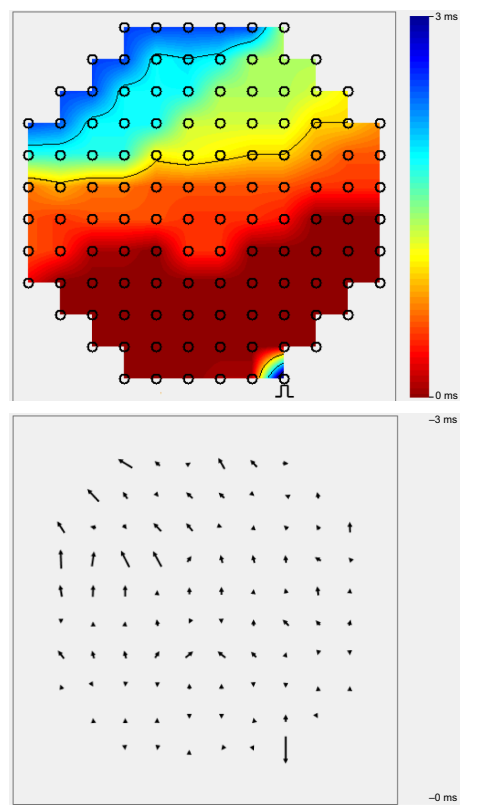

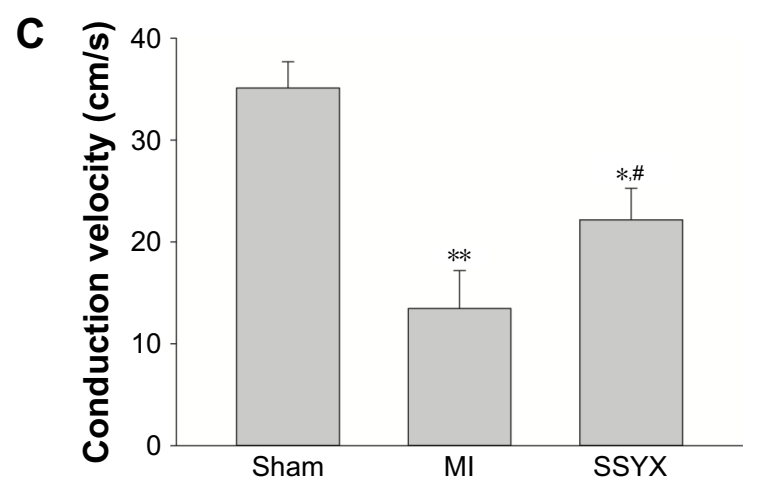

Figure 3 SSYX improved the left atrial conduction function after MI.

Notes: (A) Representative examples of the isochronous map in the left atrium by MEA recordings. Areas of isochronal crowding were found in rats with MI. The degree of crowding decreased in the SSYX-treated rats. (B) Representative examples of conduction heterogeneity map in the left atrium. Conduction was more heterogeneous in rats with MI compared to the SSYX-treated rats. (C) Bar graphs indicate conduction velocities in the three groups. SSYX improved the atrial conduction velocity after MI. $* P<0.05, * * P<0.01$ vs sham rats; ${ }^{*} P<0.05$ vs rats with MI.

Abbreviations: MEA, multielectrode array; MI, myocardial infarction; SSYX, Shensong Yangxin.

\section{Discussion}

This study investigated the effect of SSYX on chronic MIinduced atrial fibrosis and AF inducibility. The main findings of this study are as follows: 1) SSYX treatment resulted in a significant reduction in the AF inducibility rate and shortening of AF duration in rats with chronic MI; 2) SSYX treatment effectively alleviated conduction heterogeneity and improved left atrial conduction function; 3) SSYX treatment inhibited left atrial fibrosis; and 4) SSYX treatment inhibited the differentiation of cardiac fibroblasts to myofibroblasts by affecting the TGF- $\beta 1$ pathway.

$\mathrm{HF}$ and AF commonly coexist, and adversely affect mortality and increase the economic burden. ${ }^{21} \mathrm{HF}$ in patients and animal models has been shown to remodel the atrial structure, electrophysiology, and intracellular $\mathrm{Ca}^{2+}$ handling, which eventually increases susceptibility to $\mathrm{AF}^{22-25} \mathrm{HF}$ is one of the important causes of $\mathrm{AF} ;{ }^{26,27}$ therefore, we chose the MIinduced HF disease model in the present study. AF requires both a substrate and a trigger for initiation. The primary substrate is atrial remodeling (fibrosis, chamber enlargement, and atrial hypertrophy, leading to increased susceptibility to AF), whereas the primary triggers are ischemia, inflammation, and catecholamine stress. ${ }^{28-30}$ Atrial fibrosis is an important component of the arrhythmogenic substrate in patients with AF. It has been shown to increase electrical conduction disturbance and promote $\mathrm{AF}$ in different disease models. ${ }^{20,31}$ Ginsenoside Rb1 and Rg1 are the main active ingredients of ginseng, which have potential effects of attenuating liver fibrosis through the downregulation of hepatic TIMP- ${ }^{32}$ and inhibiting cardiac fibrosis through phosphor-Akt activation 

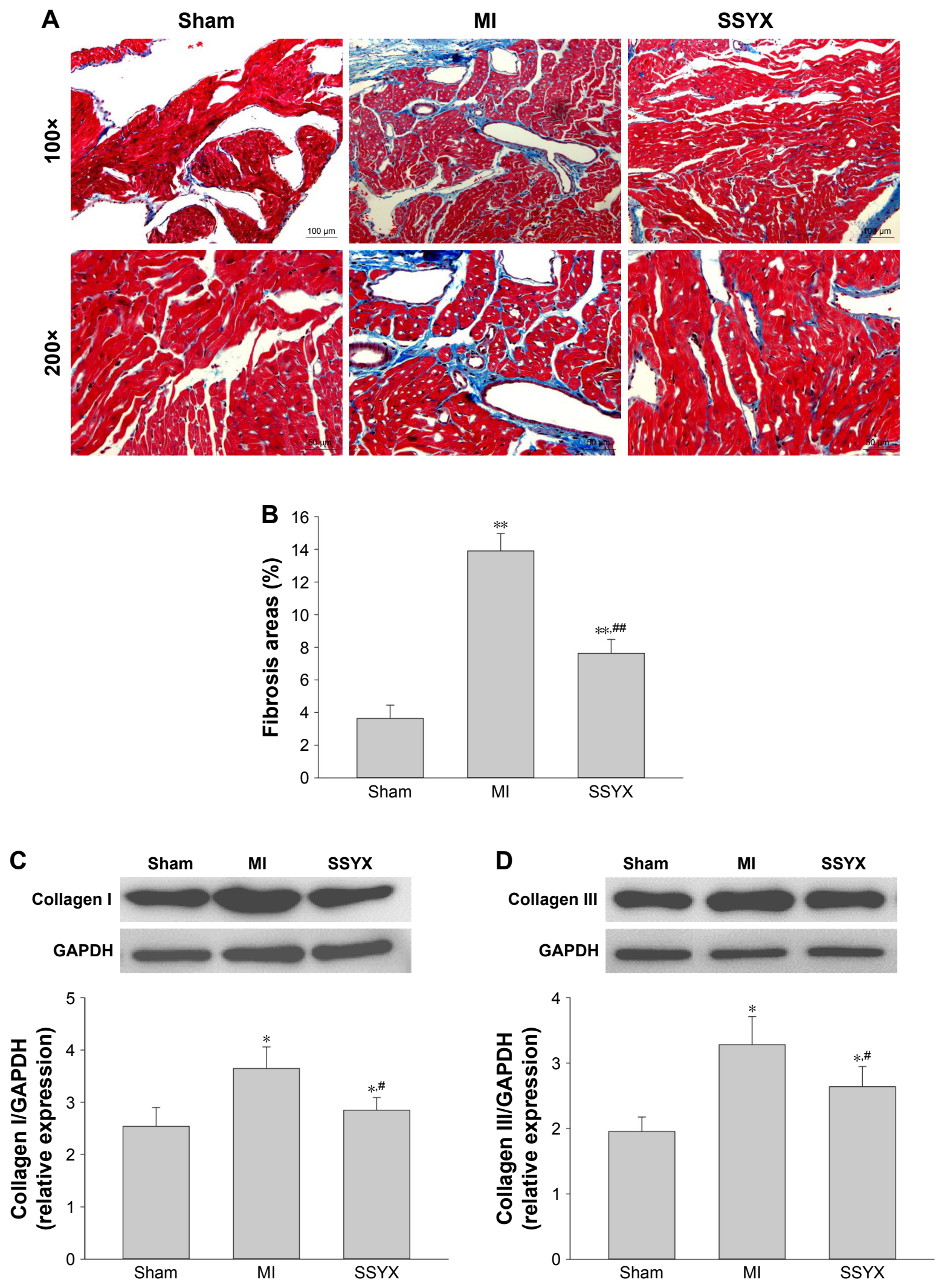

Figure 4 Effect of SSYX on left atrial fibrosis after MI.

Notes: (A) Masson's trichrome staining for the left atrial fibrosis. Fibrosis is indicated by blue areas. (B) Statistical results of left atrial fibrosis. SSYX reduced the fibrosispositive area. (C) Western blot analysis for the protein expression of collagen I. SSYX reduced the protein level of collagen I in the left atrium. (D) Western blot analysis


vs rats with MI.

Abbreviations: MI, myocardial infarction; SSYX, Shensong Yangxin. 
A


C
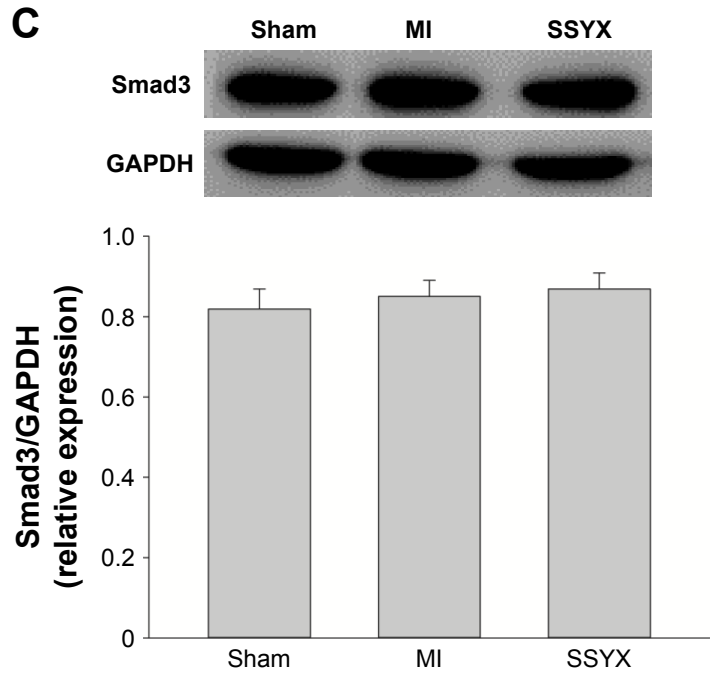
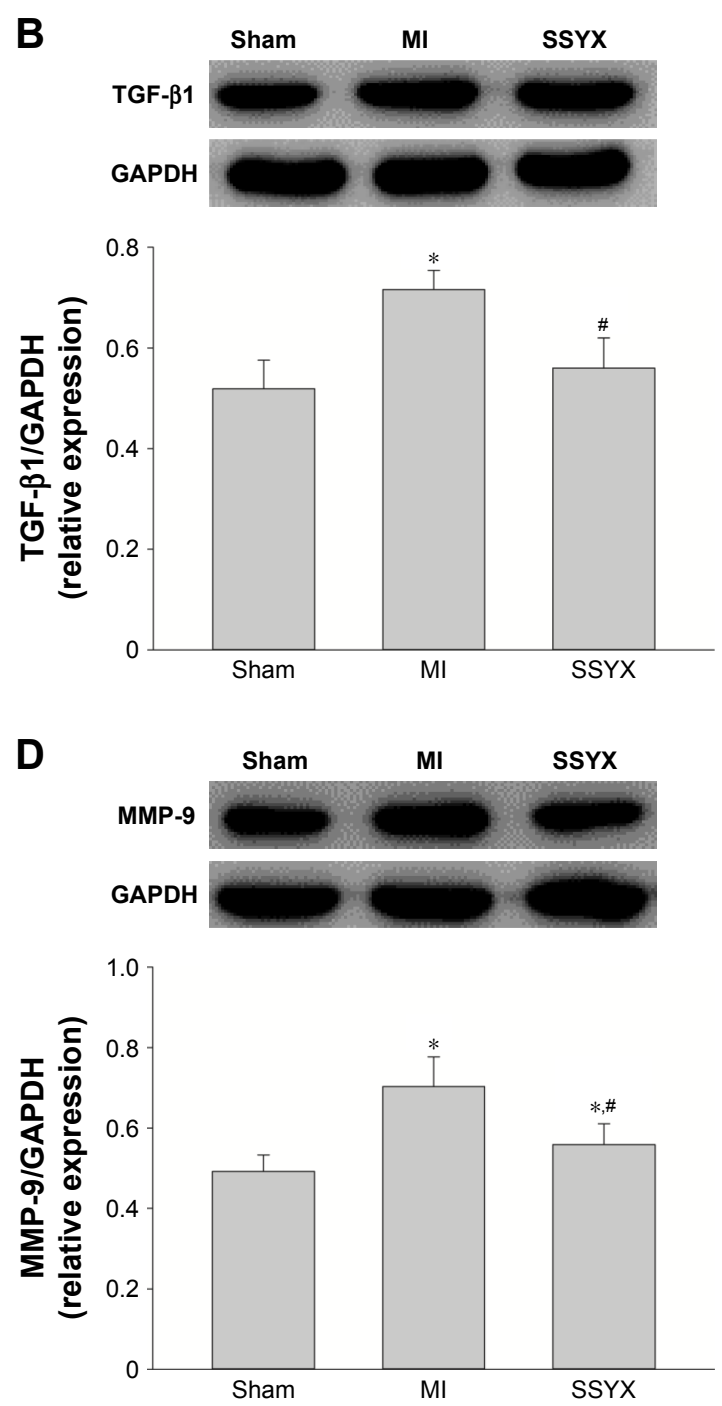

$\mathbf{E}$

MI

SSYX


Figure 5 Effect of SSYX on $\alpha$-SMA, TGF- $\beta$ I, MMP-9, and TIMP-I protein expression levels.

Notes: (A) Western blot analysis of the protein expression of $\alpha$-SMA. SSYX reduced the protein level of $\alpha$-SMA after MI. (B) Western blot analysis for the protein expression of TGF- $\beta$ I. SSYX reduced the protein level of TGF- $\beta$ I after MI $(P<0.05)$. (C) Western blots analysis of the protein expression of Smad3. SSYX had no effect on the protein level of Smad3 ( $P>0.05)$. (D) Western blot analysis of the protein expression of MMP-9. SSYX reduced the protein level of MMP-9 after MI ( $P<0.05)$. (E) Western blot analysis of the protein expression of TIMP-I. SSYX reduced the protein level of TIMP-I after MI $(P<0.05)$. ${ }^{* P}<0.05$ vs sham rats; ${ }^{\# P<0.05}$ vs rats with MI. Abbreviations: MI, myocardial infarction; SSYX, Shensong Yangxin. 
and P38 MAPK inhibition. ${ }^{33}$ The compound 7-O-galloyl-Dsedoheptulose isolated from Corni has been shown to have antifibrotic potential in diabetic mice. ${ }^{34,35}$ In addition, active compounds from Salvia have been shown to inhibit myocardial fibrosis ${ }^{36,37}$ and hepatic fibrosis through the TGF- $\beta 1$ pathway. ${ }^{38}$ SSYX contains a variety of antifibrotic components. Therefore, SSYX may have the potential to inhibit left atrial fibrosis and indirectly inhibit AF susceptibility in rats and humans with post-MI HF.

In this study, chronic MI-induced HF in rats resulted in significant left atrial fibrosis, left atrial conduction heterogeneity, and left ventricular dysfunction, and these findings are similar to the results reported in a previous study. ${ }^{39}$ However, SSYX treatment significantly attenuated atrial fibrosis, improved cardiac function, and decreased the AF inducibility rate.

Regional heterogeneity with regard to left atrial CV is an important substrate for the development of $\mathrm{AF} .{ }^{40}$ In the left atrium of patients undergoing radiofrequency ablation for AF, optical mapping revealed conduction abnormalities with different longitudinal $\mathrm{CV}$ values in different regions. ${ }^{41}$ In this study, conduction heterogeneity was noted in the left atrium of rats with MI-induced $\mathrm{HF}$, and the mean CV was lower in these rats than in sham-operated rats. Lee et al found that pirfenidone treatment in canines with HF significantly reduced not only conduction heterogeneity but also AF vulnerability to levels similar to those in controls. ${ }^{42} \mathrm{We}$ also found that the mean CV significantly improved and AF vulnerability reduced with SSYX treatment; however, AF vulnerability in SSYX-treated rats was significantly higher than that in sham-operated rats. Li et al reported that AF vulnerability reduced with enalapril treatment in the canine model of $\mathrm{HF}$, but was significantly higher than that in controls. ${ }^{43}$ Our result is consistent with this previous finding.

TGF- $\beta 1$ is a potent stimulator of collagen production through the Smad pathway, ${ }^{44}$ which is a critical pathway involved in the promotion of fibrosis. ${ }^{45}$ Kupfahl et al noted that angiotensin II may upregulate TGF- $\beta 1$ expression in human atrial tissue, and that TGF- $\beta 1$ signaling may increase collagen production. ${ }^{46}$ Overexpression of cardiac TGF- $\beta 1$ has been shown to increase atrial fibrosis, conduction heterogeneity, and AF inducibility. ${ }^{20}$ Furthermore, SSYX decreases TGF- $\beta 1$ expression in different disease models, including rats with diabetes and rabbits with chronic MI, ${ }^{17,39}$ which is consistent with our findings. In the present study, TGF- $\beta 1$ expression increased after MI, and SSYX treatment ameliorated this effect. Atrial ECM homeostasis is regulated by a balance between MMPs and TIMPs. It has been reported that elevated tissue and plasma TIMP-1 levels are correlated with myocardial fibrosis, and that TIMP-1 has been used as a biomarker for fibrosis in humans and animal models. ${ }^{47,48}$ Takawale et $\mathrm{al}^{49}$ reported that loss of TIMP-1 ameliorated myocardial fibrosis through a reduction in collagen expression, and that absence of TIMP-1 improved cardiac function in TIMP-1-deficient mice. These findings are consistent with our results. In the present study, TIMP-1 expression remarkably increased in the chronic MI rat model and significantly reduced after SSYX treatment. In addition, SSYX treatment inhibited myocardial fibrosis by reducing collagen type I and III and $\alpha$-SMA expressions. The present results suggest that SSYX attenuates AF vulnerability by attenuating atrial fibrosis, inhibiting the differentiation of fibroblasts to myofibroblasts, and improving atrial conduction.

\section{Conclusion}

AF has traditionally been treated with antiarrhythmic drugs (ion-channel blockers), which have proarrhythmic risks. Traditional Chinese medicines have been used owing to their prominent advantages, including stable curative effects and low toxicity. In the present study, SSYX reduced AF inducibility after MI by improving left atrial conduction function via inhibition of left atrial fibrosis. It also prevented the development of MI-induced vulnerable substrate for AF.

\section{Acknowledgments}

This work was supported by a grant from Natural Science Foundation of China (No 81503394), Guangzhou Science and Technology Foundation (No 201607010364), Natural Science Foundation of Guangdong Province (No 2016A030313634, No 2017A030313888), and Guangdong Province Talents Project in Colleges and Universities (No 2050205). The Specific Research Fund for TCM Science and Technology Foundation of Guangdong Provincial Hospital of Chinese Medicine (No YN2018MJ02). The abstract for this article is available online at https:// www.researchgate.net/publication/309003615 GW27e0801 Shensong Yangxin Capsule Prevents Atrial Fibrillation by Inhibiting Atrial Fibrosis in Postmyocardial Infarction Induced Heart Failure Rats.

\section{Author contributions}

Jin Ma and Chunxia Yin conceived the study. Jin Ma, Shiyu Ma, and Huiliang Qiu designed, performed, and analyzed the experiments. Chunxia Yin performed data collection and wrote the manuscript. Chaoyang Zheng performed data collection. Weihui Lv, Qiuxiong Chen, and Chunhua Ding coordinated the study and revised the manuscript. All authors contributed toward data analysis, drafting and revising the paper and agree to be accountable for all aspects of the work. 


\section{Disclosure}

The authors report no conflicts of interest in this work.

\section{References}

1. Wong CX, Brooks AG, Lau DH, et al. Factors associated with the epidemic of hospitalizations due to atrial fibrillation. Am J Cardiol. 2012;110(10):1496-1499.

2. Wong CX, Brooks AG, Leong DP, Roberts-Thomson KC, Sanders P. The increasing burden of atrial fibrillation compared with heart failure and myocardial infarction: a 15-year study of all hospitalizations in Australia. Arch Intern Med. 2012;172(9):739-741.

3. Gutierrez A, van Wagoner DR. Oxidant and inflammatory mechanisms and targeted therapy in atrial fibrillation: an update. $J$ Cardiovasc Pharmacol. 2015;66(6):523-529.

4. Thanigaimani S, Lau DH, Agbaedeng T, Elliott AD, Mahajan R, Sanders P. Molecular mechanisms of atrial fibrosis: implications for the clinic Expert Rev Cardiovasc Ther. 2017;15(4):247-256.

5. Sovari AA. Cellular and molecular mechanisms of arrhythmia by oxidative stress. Cardiol Res Pract. 2016;2016:9656078.

6. Platonov PG. Atrial fibrosis: an obligatory component of arrhythmia mechanisms in atrial fibrillation? J Geriatr Cardiol. 2017;14(4): 233-237.

7. Dzeshka MS, Lip GY, Snezhitskiy V, Shantsila E. Cardiac fibrosis in patients with atrial fibrillation: mechanisms and clinical implications. J Am Coll Cardiol. 2015;66(8):943-959.

8. Tarone G, Balligand JL, Bauersachs J, et al. Targeting myocardial remodelling to develop novel therapies for heart failure: a position paper from the Working Group on Myocardial Function of the European Society of Cardiology. Eur J Heart Fail. 2014;16(5):494-508.

9. Kawasaki M, Yamada T, Okuyama Y, et al. Eplerenone might affect atrial fibrosis in patients with hypertension. Pacing Clin Electrophysiol. 2017;40(10):1096-1102.

10. Zhao Z, Chen Y, Li W, et al. Aliskiren protecting atrial structural remodeling from rapid atrial pacing in a canine model. Naunyn Schmiedebergs Arch Pharmacol. 2016;389(8):863-871.

11. Yang $Q, W u$ G, Han L, et al. Taurine reverses atrial structural remodeling in Ach-Cacl ${ }_{2}$ induced atrial fibrillation rats. Adv Exp Med Biol. 2017;975:831-841.

12. Zou JG, Zhang J, Jia ZH, Cao KJ. Evaluation of the traditional Chinese Medicine Shensongyangxin capsule on treating premature ventricular contractions: a randomized, double-blind, controlled multicenter trial. Chin Med J (Engl). 2011;124(1):76-83.

13. Wang X, Hu D, Dang S, et al. Effects of traditional Chinese medicine shensong yangxin capsules on heart rhythm and function in congestive heart failure patients with frequent ventricular premature complexes: a randomized, double-blind, multicenter clinical trial. Chin Med J (Engl). 2017;130(14):1639-1647.

14. Zhao HY, Zhang SD, Zhang K, et al. Effect of shensong yangxin on the progression of paroxysmal atrial fibrillation is correlated with regulation of autonomic nerve activity. Chin Med J (Engl). 2017;130(2): 171-178.

15. Li N, Huo YP, Ma KJ, Sun Q, Pu JL. Effects of solution of dry powder of ShenSong YangXin capsule on sodium current and L-type calcium current in ventricular myocytes: experiment with guinea pig. Zhonghua Yi Xue Za Zhi. 2007;87(14):995-998.

16. Shi L, Yang XC, Liu XL, Zong M, Wu YL, Y1 W. Effects of ShenSongYangXin on action potential and some current channels in isolated rabbit pulmonary vein cardiomyocytes. Zhonghua Yi Xue Za Zhi. 2009; 89(30):2142-2146.

17. Shen N, Li X, Zhou T, et al. Shensong Yangxin capsule prevents diabetic myocardial fibrosis by inhibiting TGF- $\beta 1 /$ Smad signaling. J Ethnopharmacol. 2014;157:161-170.

18. Liu M, Zhao S, Wang Y, et al. Identification of multiple constituents in Chinese medicinal prescription Shensong Yangxin capsule by ultra-fast liquid chromatography combined with quadrupole time-of-flight mass spectrometry. J Chromatogr Sci. 2015;53(2):240-252.
19. Ma J, Ma S, Yin C, Wu H. Shengmai San-derived herbal prevents the development of a vulnerable substrate for atrial fibrillation in a rat model of ischemic heart failure. Biomed Pharmacother. 2018;100: $156-167$.

20. Verheule S, Sato T, Everett T, et al. Increased vulnerability to atrial fibrillation in transgenic mice with selective atrial fibrosis caused by overexpression of TGF-beta1. Circ Res. 2004;94(11):1458-1465.

21. Thihalolipavan S, Morin DP. Atrial fibrillation and heart failure: update 2015. Prog Cardiovasc Dis. 2015;58(2):126-135.

22. Kettlewell S, Burton FL, Smith GL, Workman AJ. Chronic myocardial infarction promotes atrial action potential alternans, afterdepolarizations, and fibrillation. Cardiovasc Res. 2013;99(1):215-224.

23. Sanders P, Morton JB, Davidson NC, et al. Electrical remodeling of the atria in congestive heart failure: electrophysiological and electroanatomic mapping in humans. Circulation. 2003;108(12):1461-1468.

24. Ohtani K, Yutani C, Nagata S, Koretsune Y, Hori M, Kamada T. High prevalence of atrial fibrosis in patients with dilated cardiomyopathy. J Am Coll Cardiol. 1995;25(5):1162-1169.

25. Pandit SV, Workman AJ. Atrial electrophysiological remodeling and fibrillation in heart failure. Clin Med Insights Cardiol. 2016; 10(Suppl 1):41-46.

26. King JB, Azadani PN, Suksaranjit P, et al. Left atrial fibrosis and risk of cerebrovascular and cardiovascular events in patients with atrial fibrillation. J Am Coll Cardiol. 2017;70(11):1311-1321.

27. Milliez P, Deangelis N, Rucker-Martin C, et al. Spironolactone reduces fibrosis of dilated atria during heart failure in rats with myocardial infarction. Eur Heart J. 2005;26(20):2193-2199.

28. Nishida K, Michael G, Dobrev D, Nattel S. Animal models for atrial fibrillation: clinical insights and scientific opportunities. Europace. 2010;12(2):160-172.

29. Li D, Fareh S, Leung TK, Nattel S. Promotion of atrial fibrillation by heart failure in dogs: atrial remodeling of a different sort. Circulation. 1999;100(1):87-95.

30. Workman AJ, Smith GL, Rankin AC. Mechanisms of termination and prevention of atrial fibrillation by drug therapy. Pharmacol Ther. 2011; 131(2):221-241

31. Xiao H, Lei H, Qin S, Ma K, Wang X. TGF-beta1 expression and atrial myocardium fibrosis increase in atrial fibrillation secondary to rheumatic heart disease. Clin Cardiol. 2010;33(3):149-156.

32. Hou YL, Tsai YH, Lin YH, Chao JC. Ginseng extract and ginsenoside $\mathrm{Rb} 1$ attenuate carbon tetrachloride-induced liver fibrosis in rats. BMC Complement Altern Med. 2014;14:415.

33. Zhang YJ, Zhang XL, Li MH, et al. The ginsenoside Rg1 prevents transverse aortic constriction-induced left ventricular hypertrophy and cardiac dysfunction by inhibiting fibrosis and enhancing angiogenesis. J Cardiovasc Pharmacol. 2013;62(1):50-57.

34. Park CH, Tanaka T, Yokozawa T. Evaluation of 7-O-galloyl-D-sedoheptulose, isolated from Corni Fructus, in the adipose tissue of type 2 diabetic db/db mice. Fitoterapia. 2013;89:131-142.

35. Han Y, Jung HW, Park YK. Selective therapeutic effect of Cornus officinalis fruits on the damage of different organs in STZ-induced diabetic rats. Am J Chin Med. 2014;42(5):1169-1182.

36. Mao B, Nuan L, Yang L, Zeng X. Compatibility of Astragalus and Salvia extract inhibits myocardial fibrosis and ventricular remodeling by regulation of protein kinase D1 protein. Int J Clin Exp Med. 2015; 8(3):3716-3724.

37. Zhang Y, Wang H, Cui L, et al. Continuing treatment with Salvia miltiorrhiza injection attenuates myocardial fibrosis in chronic ironoverloaded mice. PLoS One. 2015;10(4):e0124061.

38. Sferra R, Vetuschi A, Catitti V, et al. Boswellia serrata and Salvia miltiorrhiza extracts reduce DMN-induced hepatic fibrosis in mice by TGF-beta1 downregulation. Eur Rev Med Pharmacol Sci. 2012; 16(11):1484-1498.

39. Dang S, Huang CX, Wang X, Wang X, Hu J, Huang H. Shensong Yangxin (SSYX) ameliorates disordered excitation transmission by suppressing cardiac collagen hyperplasia in rabbits with chronic myocardial infarction. J Huazhong Univ Sci Technolog Med Sci. 2016; 36(2):162-167. 
40. Nattel S, Shiroshita-Takeshita A, Brundel BJ, Rivard L. Mechanisms of atrial fibrillation: lessons from animal models. Prog Cardiovasc Dis. 2005;48(1):9-28.

41. Arroja JD, Burri H, Park CI, Giraudet P, Zimmermann M. Electrophysiological abnormalities in patients with paroxysmal atrial fibrillation in the absence of overt structural heart disease. Indian Pacing Electrophysiol J. 2016;16(5):152-156.

42. Lee KW, Everett TH, Rahmutula D, et al. Pirfenidone prevents the development of a vulnerable substrate for atrial fibrillation in a canine model of heart failure. Circulation. 2006;114(16):1703-1712.

43. Li D, Shinagawa K, Pang L, et al. Effects of angiotensin-converting enzyme inhibition on the development of the atrial fibrillation substrate in dogs with ventricular tachypacing-induced congestive heart failure. Circulation. 2001;104(21):2608-2614.

44. Evans RA, Tian YC, Steadman R, Phillips AO. TGF-beta1-mediated fibroblast-myofibroblast terminal differentiation-the role of Smad proteins. Exp Cell Res. 2003;282(2):90-100.

45. Goudis CA, Kallergis EM, Vardas PE. Extracellular matrix alterations in the atria: insights into the mechanisms and perpetuation of atrial fibrillation. Europace. 2012;14(5):623-630.
46. Kupfahl C, Pink D, Friedrich K, et al. Angiotensin II directly increases transforming growth factor beta 1 and osteopontin and indirectly affects collagen mRNA expression in the human heart. Cardiovasc Res. 2000; 46(3):463-475.

47. Lindsay MM, Maxwell P, Dunn FG. TIMP-1: a marker of left ventricular diastolic dysfunction and fibrosis in hypertension. Hypertension. 2002;40(2):136-141.

48. Mukherjee R, Herron AR, Lowry AS, et al. Selective induction of matrix metalloproteinases and tissue inhibitor of metalloproteinases in atrial and ventricular myocardium in patients with atrial fibrillation. Am J Cardiol. 2006;97(4):532-537.

49. Takawale A, Zhang P, Patel VB, Wang X, Oudit G, Kassiri Z. Tissue inhibitor of matrix metalloproteinase-1 promotes myocardial fibrosis by mediating CD63-integrin $\beta 1$ interaction. Hypertension. 2017; 69(6):1092-1103.
Drug Design, Development and Therapy

\section{Publish your work in this journal}

Drug Design, Development and Therapy is an international, peerreviewed open-access journal that spans the spectrum of drug design and development through to clinical applications. Clinical outcomes, patient safety, and programs for the development and effective, safe, and sustained use of medicines are the features of the journal, which

\section{Dovepress}

has also been accepted for indexing on PubMed Central. The manuscript management system is completely online and includes a very quick and fair peer-review system, which is all easy to use. Visit http://www.dovepress.com/testimonials.php to read real quotes from published authors.

Submit your manuscript here: http://www.dovepress.com/drug-design-development-and-therapy-journal 